Política y Sociedad

ISSN: $\quad 1130-8001$

ISSN-e: $1988-3129$

\title{
Gobiernos autonómicos españoles y transparencia activa de sus repositorios digitales. Una revisión taxonómica
}

\author{
Ricardo Curto-Rodríguez ${ }^{1}$
}

Recibido: 02-03-2020 / Aceptado: 29-07-2020

Resumen. Las administraciones públicas suministran datos sobre su desempeño implementando los principios del gobierno abierto. Con el doble objetivo de cuantificar la divulgación proactiva de información digital de las comunidades autónomas españolas en diciembre de 2013 y de 2017, así como buscar tipologías, nos serviremos de una lista de comprobación propia inspirada en la Ley nacional 19/2013 de transparencia, acceso a la información pública y buen gobierno. Esta, al ponderar su facilidad reutilizadora según la escala cinco estrellas de Tim Berners-Lee, nos permitirá formular dos indicadores: transparencia open data, que recoge únicamente la información de los portales de datos abiertos, y transparencia global, que amplía la búsqueda a los portales de transparencia y a la web institucional. Los resultados muestran que los niveles de divulgación dejan bastante que desear y, aunque existe una ligera mejoría con el paso del tiempo, solo es relevante para transparencia global gracias al desarrollo y aportación de los portales de transparencia que eclipsan la utilidad de la web institucional. El análisis tipológico identifica dos conglomerados bastante equilibrados, y da soporte al postulado de dos teorías contrapuestas: agencia y neoinstitucional.

Palabras clave: análisis clúster; transparencia; datos abiertos; comunidades autónomas; España.

\section{[en] Spanish Autonomous Governments and Active Transsparency of their Digital Repositories. A Taxonomic Review}

\begin{abstract}
Public administrations provide data about their performance, implementing the open government principles. With the aim of both quantifying the proactive disclosure of digital information from the Spanish Autonomous Communities in december 2013 and 2017, as well as looking for typologies, we will be using a self verification list inspired on the national law 19/2013 about transparency, access to public information and good government, which will assign a weight according to Tim Berners-Lee's five star scale, and will allow us to formulate two indicators: open data transparency, which only gathers information from the open data websites, and global transparency, which expands the search to the transparency portals and the institutional web site. The outcome of the investigation shows that the disclosure levels are unsatisfactory overall and, although they have improved lately, they are only relevant for global transparency thanks to the development and contribution of the transparency portals, which overshadow the usefulness of the institutional web site. The typological analysis identifies two balanced conglomerates supporting the postulate of two opposing theories: agency and neo-institutional.
\end{abstract}

Keywords: cluster analysis; transparency; open data; regional goverments; Spain.

Universidad de Oviedo (España).

E-mail: curtoricardo@uniovi.es 
Cómo citar: Curto-Rodríguez, R. (2020): “Gobiernos autonómicos españoles y transparencia activa de sus repositorios digitales. Una revisión taxonómica”, Política y Sociedad, 57(2), pp. 543-565.

Sumario. 1. Introducción. 2. Metodología. 3. Resultados. 4. Conclusiones, limitaciones y futuras líneas de investigación. 5. Bibliografía.

\section{Introducción}

A partir de la formulación original de gobierno abierto u open goverment de Parks (1957), que recomendaba facilitar el acceso a los datos en poder de las administraciones públicas, se ha ido configurando el objetivo de "abrir las ventanas" del sector público (Chapman y Hunt, 1987) dejando que la luz del sol actúe como desinfectante (Cordis y Warren, 2014).

Actualmente, la definición de gobierno abierto ha evolucionado: "Gobierno abierto es aquel que entabla una constante conversación con los ciudadanos con el fin de oír lo que ellos dicen y solicitan, que toma decisiones basadas en sus necesidades y preferencias, que facilita la colaboración de los ciudadanos y funcionarios en el desarrollo de los servicios que presta y que comunica todo lo que decide y hace de forma abierta y transparente" (Calderón y Lorenzo, 2010), incorporando los tres pilares configuradores mencionados por Obama (2009): transparencia, participación y colaboración.

Fue el propio Obama quien fomentara el enfoque digital, redescubriendo el concepto (Conejero, 2013) y consolidándolo como una alternativa al paradigma de gobierno anteriormente utilizado (Villoria Mendieta 2012, 2013). El nacimiento de la web 2.0 tuvo mucho que ver en dicho impulso (Cotino, 2013; Criado, 2013; Mariñez Navarro, 2103, 2017), al permitir la masiva puesta en práctica del open goverment con unos costes asumibles para los Estados (Cobo, 2013). Y es que la red ha transformado de manera estructural la esfera pública con espacios de participación abierta y gratuita (Masip et al., 2019), facilitando el imparable ${ }^{2}$ desarrollo de los open data que, al fomentar los formatos digitales, estandarizados, abiertos y reutilizables (Giménez-Chornet, 2012), favorece el desarrollo del gobierno abierto.

La apertura de los datos públicos posibilita la producción de nuevos productos y servicios, pero también permite conocer, analizar y evaluar el desempeño gubernamental, la transparencia (Cerrillo-Martínez, 2014), que puede contribuir a generar una mejora de la confianza en el gobierno (Reddick, 2011) y favorecer la rendición de cuentas (Bauhr y Grimes, 2012)

Es indudable que en una democracia avanzada debe existir un flujo libre y acceso a la información pública sobre el Gobierno (Krah y Mertens, 2020). Si bien es cierto que la transparencia tiene su punto de partida en la divulgación de información en manos de los Gobiernos, y que este suministro de datos debe verse como algo natural al estar financiando la ciudadanía los fondos que manejan los gestores públicos (Bakar y Saleh, 2011; Campos Acuña y Vaquero García, 2019), serán las nuevas técnicas de minería de datos, big data y las tecnologías de

Recientemente ha sido aprobada una nueva directiva europea sobre datos abiertos y reutilización de la información del sector público (Directiva UE 2019/1024), de 20 de junio de 2019, que estimula la innovación digital y el acceso en tiempo real a los datos dinámicos. Los países miembros tienen de plazo hasta el 17 de julio de 2021 para transponer la directiva a su ordenamiento nacional. 
procesamiento las que permitan valorar la eficacia de las políticas públicas (Boix, 2015; Cerrillo-Martínez, 2018).

Los open (government) data ${ }^{3}$ se han convertido en un signo inequívoco de una administración transparente y responsable (Höchtl, 2012). Y es que "los datos" son importantes en nuestra Ley 19/2013 de transparencia, acceso a la información pública y buen gobierno (Beltrán-Orenes y Martínez-Pastor, 2016), norma consecuencia de la inercia histórica, política, social y, por supuesto, jurídica de nuestro entorno (Cotino Hueso, 2015a). Con su aprobación ha permitido a España dejar de ser el único país miembro del Consejo de Europa con más de 1 millón de habitantes que no disponía de una ley específica de transparencia y acceso a la información pública (Villoria Mendieta, 2014).

Esta ley ${ }^{4}$, que respondía a los compromisos adquiridos con la alianza por el gobierno abierto (open government partnership), ha sido calificada como controvertida, provocando una división entre quienes afirman que cumplirá el objetivo de cambiar las actitudes políticas y contribuirá a la regeneración democrática, y aquellos que la califican como descafeinada y poco ambiciosa. Su relevancia es indiscutible, al igual que otra serie de iniciativas que pretenden igualmente fomentar el desarrollo del gobierno abierto: comunicaciones, reglamentos, directivas, proyectos, portales o normas técnicas, que se pueden consultar en Curto-Rodríguez (2017).

Nos detendremos en las de ámbito regional español, objeto de nuestro estudio. Hemos elegido ese nivel gubernamental, apenas explorado por la literatura, al ser España un Estado cuasifederal (Bastida et al., 2019) donde las comunidades autónomas (CC. AA.) tienen transferidas competencias muy relacionadas con los servicios básicos de un Estado de bienestar, como los servicios sociales, la sanidad o la educación, de especial interés para la ciudadanía (Fernández Llera y Morán Méndez, 2013).

Hemos dividido las iniciativas en dos categorías. Por un lado, estarían tres tipos de canales de divulgación de información: web institucional (WI), website de carácter generalista en el que una determinada administración ofrece contenido de cualquier tipo (primer canal empleado para el suministro de información pública); portal de transparencia (PT), repositorio específico para depositar información relevante para la rendición de cuentas (prima como objetivo la facilidad de visualización frente a la reutilización, donde abunda el empleo de los formatos pdf); y portal de datos abiertos (POD), repositorio web que contiene conjuntos de datos o datasets que fomentan su reutilización al ofertar, generalmente, licencias libres y formatos abiertos (la tabla 1 muestra información sobre su estado y su reciente evolución).

\footnotetext{
3 En la nota al pie "forma temprana Curto-Rodríguez", falta algo en la redacción? España se ha sumado exitosamente a los datos abiertos de forma temprana Curto-Rodríguez (2015), siendo un referente de buenas prácticas y uno de los líderes europeos según varios estudios internacionales recogidos en Curto-Rodríguez (2020).

4 No pretendemos, ni es posible por limitaciones de espacio, ser exhaustivos en el comentario de esta ley. Remitimos a los trabajos de afamados expertos en derecho para profundizar en su comprensión y análisis: Guichot Reina, (2014); Sánchez de Diego Fernández de La Riva, (2014); Cotino Hueso (2015b); Delgado Morales et al. (2015) y Sierra Rodríguez, (2018a).
} 
Tabla 1. Portales de datos abiertos autonómicos y conjuntos de datos albergados

\begin{tabular}{|c|c|c|c|c|}
\hline Comunidad autónoma & Nombre del portal & \begin{tabular}{|c|} 
Datasets \\
diciembre 2013 \\
\end{tabular} & $\begin{array}{c}\text { Datasets } \\
\text { diciembre } 2015 \\
\end{array}$ & \begin{tabular}{|c|} 
Datasets \\
diciembre 2017 \\
\end{tabular} \\
\hline Andalucía & Datos Abiertos & 70 & 178 & 464 \\
\hline Aragón & Aragón Open data & 440 & 2519 & 2794 \\
\hline Canarias & Datos Abiertos Canarias & Sin Portal & 45 & 43 \\
\hline Cantabria & & Sin portal & Sin portal & Sin portal \\
\hline Castilla y León & Datos Abiertos de Castilla y León & 124 & 176 & 334 \\
\hline Castilla-La Mancha & Datos Abiertos de Castilla- La Mancha & 42 & 59 & 207 \\
\hline Cataluña & Datos Abiertos GenCat & 1460 & 1468 & 571 \\
\hline Comunidad de Madrid & & Sin portal & Sin portal & Sin portal \\
\hline Com. Foral de Navarra & Open data & 135 & 190 & 1186 \\
\hline Comunidad Valenciana & Dades obertes gva & Sin portal & 279 & 338 \\
\hline Extremadura & Gobierno Abierto - Catálogo de datos & 26 & 25 & 25 \\
\hline Galicia & abert@s & 292 & 324 & 349 \\
\hline Islas Baleares & Dades obertes caib & 42 & 42 & 42 \\
\hline La Rioja & Datos Abiertos Rioja & 64 & 86 & 265 \\
\hline País Vasco & Opendataeuskadi & 2280 & 2242 & 4123 \\
\hline Principado de Asturias & Open data del Principado de Asturias & Sin portal & 419 & 42 \\
\hline Región de Murcia & Datos abiertos Región de Murcia & Sin portal & Sin portal & 271 \\
\hline
\end{tabular}

Fuente: elaboración propia.

Por otro lado, estarían el resto de iniciativas. Alcaide Muñoz et al. (2019) recogen las que mostramos en la tabla 2, (una vez excluidos los proyectos de datos abiertos que terminan de ser ofrecidos con mayor detalle).

No esperamos encontrar uniformidad en la divulgación autonómica. Un motivo es que las CC. AA. españolas son muy diferentes entre sí (población, superficie, nivel de renta, identidad histórica, lingüística y cultural, etc.). El otro motivo se fundamenta en lo sugerido por dos teorías y modelos de comportamientos (contrapuestas/tos) que pretenden explicar el grado de suministro de información pública.

Comenzamos con la teoría de la agencia, la más empleada en la literatura (Rodríguez Bolívar et al., 2013). Se aplica en situaciones donde unos individuos asignan atribuciones a otros para actuar en su nombre. En el ámbito de la empresa, el "principal" son los propietarios, que encargan a los "agentes" (administrador o gestor), la defensa de sus intereses, delegando en ellos cierto poder de decisión. En el ámbito de la Administración pública, los Gobiernos serían los "agentes" mientras que los gobernados sería el "principal", cuyo interés reside en evitar que el agente obre en su propio favor apartándose de los deseos y necesidades de la ciudadanía. La transparencia, además de uno de los factores determinantes en la lucha contra la corrupción (Lizcano, 2016), es esencial para superar la asimetría informativa entre políticos y ciudadanos (Esteller-Moré y Polo, 2012), ya que una mayor divulgación puede permitir el monitoreo de los gobernantes (Laswad, Fisher y Oyerele, 2005) resolviendo el problema de la agencia (Ferejohn, 1999). 
Tabla 2. Resto de iniciativas de gobierno abierto desarrolladas por las autonomías

\begin{tabular}{|c|c|}
\hline Comunidad & Planes, actuaciones y regulaciones de gobierno abierto \\
\hline Andalucía & $\begin{array}{ll} & \text { Plan de Gobierno Abierto (2018-2019) } \\
\square \quad \text { Ley } 1 / 2014 \text {, de } 24 \text { de junio de Transparencia Pública de Andalucía }\end{array}$ \\
\hline Aragón & $\begin{array}{ll} & \text { Programa anual de participación ciudadana } \\
\square \quad \text { Ley } 8 / 2015 \text {, de } 25 \text { de marzo, de Transparencia de la Actividad Pública y Participación } \\
\text { Ciudadana de Aragón. }\end{array}$ \\
\hline Canarias & $\square \quad$ Estrategia de gobierno abierto (2017-2019) \\
\hline Cantabria & $\square \quad$ Ley $1 / 2018$, de 21 de marzo, de Transparencia de la Actividad Pública \\
\hline $\begin{array}{l}\text { Castilla y } \\
\text { León }\end{array}$ & $\begin{array}{l}\square \quad \text { Acuerdo } 17 / 2012 \text { sobre modelo de gobierno abierto } \\
\square \quad \text { Ley 3/2015, de } 4 \text { de marzo, de Transparencia y Participación Ciudadana de Castilla y } \\
\text { León }\end{array}$ \\
\hline $\begin{array}{l}\text { Castilla-La } \\
\text { Mancha }\end{array}$ & $\begin{array}{l}\square \quad \text { Proyecto de ley de participación (2018) } \\
\square \quad \text { Ley 4/2016, de } 15 \text { de diciembre, de Transparencia y Buen Gobierno de Castilla-La } \\
\text { Mancha }\end{array}$ \\
\hline Cataluña & $\begin{array}{l}\square \quad \text { Plan de Gobierno Abierto (2017-2020) } \\
\square \quad \text { Ley 19/2014, de } 29 \text { de diciembre, de Transparencia, Acceso a la Información Pública y } \\
\text { Buen Gobierno }\end{array}$ \\
\hline $\begin{array}{l}\text { Comunidad } \\
\text { de Madrid }\end{array}$ & $\begin{array}{ll} & \text { Plan de acción de gobierno (2015-2019) } \\
\square \quad \text { Ley } 19 / 2013 \text {, de } 9 \text { de diciembre, de Transparencia, Acceso a la Información pública y } \\
\text { Buen Gobierno }\end{array}$ \\
\hline $\begin{array}{l}\text { Comunidad } \\
\text { Foral de } \\
\text { Navarra }\end{array}$ & $\begin{array}{ll} & \text { Plan para impulsar la participación ciudadana (2017-2019) } \\
\square \quad \text { Plan normativo anual } 2018 \\
\square \quad \text { Ley foral 5/2018, de } 17 \text { de mayo, de Transparencia, Acceso a la Información Pública y } \\
\text { Buen Gobierno }\end{array}$ \\
\hline $\begin{array}{l}\text { Comunidad } \\
\text { Valenciana }\end{array}$ & $\begin{array}{l}\square \quad \text { Plan estratégico de gobierno abierto (2016-2019) } \\
\square \quad \text { Ley } 2 / 2015 \text {, de } 2 \text { de abril, de Transparencia, Buen Gobierno y Participación Ciudadana } \\
\text { de la Comunitat Valenciana }\end{array}$ \\
\hline Extremadura & $\begin{array}{l}\square \quad \text { Plan de Alfabetización tecnológica (2015-2019) } \\
\square \quad \text { Ley 4/2013, de } 21 \text { de mayo, de Gobierno Abierto de Extremadura }\end{array}$ \\
\hline Galicia & $\begin{array}{l}\square \quad \text { Programa de impulso democrático (2015-2016) } \\
\square \quad \text { Ley } 1 / 2016 \text {, de } 18 \text { de enero, de Transparencia y Buen Gobierno }\end{array}$ \\
\hline $\begin{array}{l}\text { Islas } \\
\text { Baleares }\end{array}$ & $\begin{array}{ll}\square & \text { Plan normativo anual } 2018 \\
\square & \text { Ley 4/2011, de } 31 \text { de marzo, de la Buena Administración y del Buen Gobierno de las } \\
& \text { Illes Balears }\end{array}$ \\
\hline La Rioja & $\begin{array}{l}\square \quad \text { Agenda digital (2015-2020) } \\
\square \quad \text { Ley } 3 / 2014 \text {, de } 11 \text { de septiembre, de Transparencia y Buen Gobierno de La Rioja }\end{array}$ \\
\hline País Vasco & $\begin{array}{ll} & \text { Euskadi.eus (información, procedimientos y servicios del gobierno vasco) } \\
\square & \text { Irekia (web dedicada al gobierno abierto) } \\
\square & \text { Proyecto de Ley } 2016 \text { del País Vasco sobre Transparencia y Buen Gobierno } \\
\square & \text { Plan de acción de gobierno (2015-2019) }\end{array}$ \\
\hline $\begin{array}{l}\text { Principado } \\
\text { de Asturias }\end{array}$ & $\begin{array}{ll}\square & \text { Plan estratégico de transparencia } \\
\square & \text { Libro Blanco de participación ciudadana } \\
\square \quad \text { Proyecto de Ley } 2016 \text { del Principado de Asturias sobre Transparencia y Buen gobierno }\end{array}$ \\
\hline $\begin{array}{l}\text { Región de } \\
\text { Murcia }\end{array}$ & $\begin{array}{ll}\square \quad \text { Plan de Acción de gobierno abierto (2018-2019) } \\
\square \quad \text { Ley 12/2014, de } 16 \text { de diciembre, de Transparencia y Participación Ciudadana de la } \\
\text { Comunidad Autónoma de la Región de Murcia } \\
\square \quad \text { Estrategia de gobierno abierto (2017-2019) }\end{array}$ \\
\hline
\end{tabular}

Fuente: adaptado de Alcaide Muñoz et al. (2019). 
En segundo lugar, se encuentra la teoría neoinstitucional, que sostiene que la puesta a disposición de información se asocia con un Gobierno moderno, reputado y con mejor percepción ciudadana. En base a este principio, observamos que las Administraciones públicas no dudan en replicar iniciativas que contribuyan a una mejor opinión pública, como son los portales de datos abiertos o de transparencia, en un claro ejercicio de isomorfismo institucional (García-García y Curto-Rodríguez, 2019). No obstante, publican información irrelevante para el control de su gestión y evitan, por tanto, la supervisión ciudadana en cuanto al destino de los recursos públicos.

A partir de estas teorías, los comportamientos esperados de las CC. AA. españolas serían dos (y diametralmente opuestos): ofertar abundante información relacionada con la transparencia activa, si siguen lo propuesto por la teoría de la agencia, o escasa o nula información si actúan según lo que recoge la teoría neoinstitucional.

\section{Metodología}

La transparencia es una especie de vitrina donde el actuar gubernamental puede estar bajo el escrutinio público (Gómez McFarland, 2016). Se trata de un valor en alza en la gestión pública, un principio de buena gestión y una exigencia legal (Fernández Llera, 2015) y, aunque la literatura ha aportado tantas definiciones de transparencia como intentos ha habido para definirla (Cerrillo-i-Martínez, 2012), es difícil calcular una magnitud que la represente (Bellod-Redondo, 2008).

No obstante, hay unos mínimos de contenido a considerar que se corresponden con la publicidad activa ${ }^{5}$ que establece la Ley 19/2013 de transparencia, acceso a la información pública y buen gobierno en sus artículos 6 a 8 (Sierra Rodríguez, 2018b). A partir de la observación de los artículos 6 (información institucional, organizativa y de planificación) y 8 (información económica, presupuestaria y estadística), hemos creado una lista de comprobación compuesta por 20 ítems para evaluar cuántos de ellos son atendidos en formato digital, con información completa y de forma actualizada (tabla 3 ).

La atención de cada uno de los ítems va a aportar un punto al indicador de transparencia. En todo caso, dado que entendemos que la puesta a disposición de la información debe cumplir unos criterios de accesibilidad para poder ser reutilizada (Martínez Moya, 2015), se ponderará el formato en que se suministra la información según la escala de 5 estrellas (Berners-Lee, 2010).

De esta manera, si el formato pertenece al nivel 1 estrella, es decir, es "no estructurado" (ejemplo: pdf) se otorgará un punto adicional; dos puntos si es clasificado como nivel 2 estrellas, formado por los formatos propietarios legibles por máquina (ejemplo: doc); tres puntos si perteneciera el nivel 3 estrellas, compuesto por los datos que están en formato estructurado y son no propietarios (ejemplo: csv), y cuatro puntos si son datos vinculables y reutilizables que permitan ser enlazados con otros (propios de la web semántica- niveles 4 y 5 estrellas), como por ejemplo los rdf.

Se refiere a la información que las entidades públicas o privadas deben hacer pública en sus sitios web ad hoc. La publicidad pasiva (derecho a la información) permite, al amparo de dicha ley, solicitar determinada información que no está publicada, exceptuando los casos recogidos en la misma (Beltrán-Orenes y Martínez-Pastor, 2019). 
Así pues, completar los 20 ítems de información buscada en formatos asociados a los estándares de la web semántica permitiría alcanzar los 100 puntos. El primer indicador formulado es denominado transparencia open data (TOD), y se limita a valorar el contenido de los portales de open data, mientras que el segundo indicador recibe el nombre de trasparencia global (TG) y amplía la búsqueda de información a los portales de transparencia y de la web institucional (por ese orden).

Tabla 3. Lista de comprobación

\begin{tabular}{|c|c|}
\hline Rendición de cuentas económico-financiera \\
\hline Presupuestos de gastos e ingresos: \\
$\checkmark \quad$ Por cualquier clasificación (orgánica, funcional o económica) \\
$\checkmark \quad$ Por partidas presupuestarias \\
Ejecución presupuestaria: \\
$\checkmark \quad$ Información mensual de ejecución \\
$\checkmark \quad$ Liquidación anual \\
$\checkmark \quad$ Modificaciones presupuestarias \\
Presupuestos de entes, sociedades y fundaciones públicas \\
Cuentas anuales de empresas públicas \\
Cuenta General de la Comunidad Autónoma \\
Cumplimiento objetivo de estabilidad presupuestaria \\
Nivel y desglose de endeudamiento \\
Informe de fiscalización \\
\hline Rendición de cuentas de los gobernantes \\
\hline Organigrama y contacto \\
Retribuciones a miembros de gobierno y altos cargos \\
Declaraciones de bienes de los miembros de gobierno \\
Declaraciones actividades de miembros de gobierno \\
Lista y retribuciones de cargos de confianza \\
\hline Rendición de cuentas en las ayudas y contrataciones \\
\hline Ayudas y subvenciones \\
Becas, premios o concursos \\
Contratos adjudicados \\
\hline
\end{tabular}

Fuente: elaboración propia.

Una vez presentados los indicadores que serán utilizados en el estudio procede, en este momento, realizar la fundamentación teórica y la descripción de los métodos empleados para realizar una clasificación taxonómica de las autonomías españolas; nos estamos refiriendo al análisis clúster. 
Los investigadores se encuentran a menudo con situaciones cuya mejor forma de resolución es definir grupos de objetos, empresas, individuos, productos o incluso comportamientos (Hair et al., 1999: 491). Tres son las tres técnicas estadísticas que pueden emplearse para analizar la pertenencia de casos a diferentes grupos: análisis clúster, análisis discriminante y análisis de segmentaciones (Martín Martín y de Paz Santana, 2007: 294) siendo, de todos ellos, el clúster ${ }^{6}$ el más utilizado.

Este análisis clúster, también denominado análisis de conglomerados, análisis tipológico, taxonomía numérica o de clasificación automática (Valderrey, 2010: 359) es el nombre genérico con el que son designados los más de 100 métodos utilizados para intentar encontrar conglomerados dentro de unos datos iniciales (Lévy y Varela Mallou, 2003: 420). Dos son los objetivos perseguidos: por un lado, que los grupos sean homogéneos, es decir, que cada observación que compone el grupo sea similar al resto de individuos que lo conforman y, por otro, que los grupos sean lo más diferentes posibles entre sí (Uriel Jiménez y Aldás Manzano, 2005: 48; Ferrán Aranaz, 1996: 395-396).

La puesta en marcha de la técnica comienza con la consideración de una o más variables segmentadoras para realizar los conglomerados. La mayor parte de las veces se tiende a tomar un número elevado de variables, lo que suele causar problemas en algunos métodos al aumentar exponencialmente el número de cálculos (Lévy y Varela Mallou, 2003: 421). Por ello hemos decido emplear una única variable consiguiendo una obtención de grupos aparentemente sencilla (Abascal Fernández y Grande Esteban, 2007: 397), pero con todas las bondades de cualquier proceso estadístico: minimización del sesgo humano e imparcialidad en los resultados. La variable seleccionada es la puntuación autonómica alcanzada en el indicador de transparencia (bien sea open data o global).

Como se ha mencionado anteriormente, la existencia de múltiples posibilidades hace que la elección del método para llevar a cabo el análisis clúster no sea baladí. La mayoría de autores recomienda utilizar diversos procedimientos y comparar sus resultados (Sharma, 1996; Johnson, 1998). En concreto, lo más razonable es abordar dos etapas, una primera utilizando métodos jerárquicos, lo que nos va a dar una aproximación lógica del número de conglomerados existentes $\mathrm{y}$, posteriormente, una no jerárquica que va a maximizar la homogeneidad intragrupos y la heterogeneidad intergrupos (Uriel Jiménez y Aldás Manzano, 2005: 85), que recordemos es el objetivo de todo análisis clúster.

Emplearemos, en primer lugar, un método jerárquico (muy recomendable cuando hay pocos individuos) de carácter descendiente (procedimiento más utilizado) que da comienzo con tantos grupos como individuos $\mathrm{y}$, paso a paso, va formando conglomerados hasta terminar con un solo grupo que incluye a todos los individuos. Evidentemente, el investigador deberá detener la secuencia de formación de conglomerados en un momento previo a la finalización de la agrupación, fijando para ello un determinado criterio de parada (Fariña Gómez et al., 1996).

Para una mayor profundización en el estudio de las técnicas clúster se puede consultar el monográfico "Cluster Análysis" de Everitt et al., (2001). 
En este estudio nos apoyaremos en la representación gráfica de los resultados o dendograma ${ }^{7}$ para interrumpir la formación de los clústeres cortando las ramas donde estas sean más largas, es decir, en aquel momento en el que se procedería a unir individuos más diferentes. Nos hemos decantado por el criterio formulado por Ward (1963) denominado método de mínima varianza de Ward, ya que su proceso de cálculo generalmente es el que se adapta mejor a los objetivos de la investigación (Trespalacios Gutiérrez et al., 2016: 244), al crear grupos más compactos y de tamaño similar, siendo además poco sensible a los outliers (Salvador Figueras, 2001; Luque, 2000: 164).

En segundo lugar, utilizaremos un método de tipo no jerárquico que divide una población de $n$ objetos en $k$ grupos con un criterio de optimización global (siendo imprescindible decidir a priori el número de conglomerados que deseamos como solución). La técnica elegida ha sido creada por Anderberg (1973), y se la conoce como $k$ means o algoritmo de refinamiento iterativo, estando $k$ guiada por lo observado en el método jerárquico según recomiendan Trespalacios Gutiérrez et al., (2005: 260; 2016: 247). Esta forma de actuar nos permitirá valorar la robustez de una propuesta de tipo jerárquico, que suele producir resultados más ricos que las calculadas por los métodos no jerárquicos (Pérez López; 2005: 460), que presentan una estructura matemática menos precisa (Valderrey, 2010: 364).

Una vez ejecutados los análisis clúster, el proceso finaliza verificando la adecuación del modelo y comprobando que la solución obtenida sea coherente (Martínez Miranda, 2000). Por ello, tras la discusión metodológica procede su aplicación, que va a permitir presentar los principales hallazgos obtenidos a los que se destina el siguiente apartado que ofrece los cálculos y representaciones gráficas realizadas con el programa informático SPSS versión 23.

\section{Resultados}

Los resultados son expuestos de forma cronológica; comienzan con un estudio descriptivo y un diagrama de barras, seguido de un análisis tipológico individualizado para los indicadores TOD y TG empleando, cuando resulta necesario, los métodos de Ward y $k$-means.

\subsection{Resultados diciembre 2013}

La visita a los repositorios se llevó a cabo el último mes de 2013. A principios de 2014 se realizó una revisión de los datos, y se procedió a una nueva recogida si se superaba nuestro límite de tolerancia (3\% de los datos iniciales y/o 25 datasets en el caso de los portales de datos abiertos).

En esa fecha ya eran 11 las CC. AA. que habían abierto su POD, pero dado que tres no albergaban ninguno de los ítems buscados (Galicia, Castilla-La Mancha y La Rioja), las autonomías con puntuaciones positivas en el indicador TOD quedaban reducidas a ocho. También eran 11 las CC. AA. con PT en

Abascal Fernández y Grande Estaban (1994) muestran numerosos casos de completas investigaciones comerciales reales empleando, en seis ocasiones, dendogramas con detalladas explicaciones que van desde la construcción de la propia representación gráfica, al número de conglomerados a seleccionar y a las principales conclusiones a extraer. Se trata de una obra de gran interés para familiarizarse con el análisis clúster de forma eminentemente práctica. 
funcionamiento, aunque como Andalucía no ofrecía ningún ítem que no estuviera ya disponible en el portal de datos abiertos, dejó en diez el número de CC. AA. con puntuaciones superiores a cero en dicho repositorio. Respecto a la web autonómica, 13 son las CC.AA. que aportan ítems al indicador TG, a pesar de que la mayor parte de la información se encuentra desperdigada y alojada de forma difícilmente localizable. La figura 1 muestra la representación gráfica de los resultados obtenidos y la tabla 4 el resumen.

Figura 1. Aportación a transparencia open data y transparencia global por repositorio en 2013



Fuente: elaboración propia.

La observación de la tabla 4 permite realizar interesantes comentarios. En primer lugar que, aunque el aporte en ítems de POD es prácticamente la mitad del que realizan PT o WI, las puntuaciones totales de cada iniciativa son prácticamente idénticas, gracias a la mayor calidad en los formatos que ofrecen los POD, lo que permite compensar las mencionadas diferencias en ítems atendidos.

Tabla 4. Ítems atendidos, puntos alcanzados y sus valoraciones medias 2013

\begin{tabular}{|l|c|c|c|c|}
\hline Iniciativa & Ítems atendidos & Media ítems & Puntos totalizados & Media puntos \\
\hline POD & 37 & 2,18 & 146 & 8,59 \\
\hline PT & 73 & 4,29 & 152 & 8,94 \\
\hline WI & 68 & 4,00 & 142 & 8,35 \\
\hline En conjunto & 178 & 10,47 & 440 & 25,88 \\
\hline
\end{tabular}

Fuente: elaboración propia. 
Es destacable igualmente que PT y WI tengan un desempeño aproximado, tanto en número de ítems como en puntuación total. El hecho de que bastantes autonomías carecieran de PT va a permitir calificar como relevante la labor de la web autonómica en 2013, adjetivo que no podrá mantener en 2017 una vez que todas las CC. AA. dispongan de PT. Se aprecia, además, la existencia de una correlación negativa $(r=-0,773)$ y significativa (nivel 0,01$)$ entre PT y WI, lo cual era esperado puesto que si la puntuación de PT es baja o nula, hay bastantes posibilidades de encontrar información desperdigada por la WI, mientras que cuando el PT existe y centraliza la información de transparencia activa, resulta difícil encontrar en WI información adicional.

Figura 2. Dendograma transparencia global 2013, clúster jerárquico método de Ward

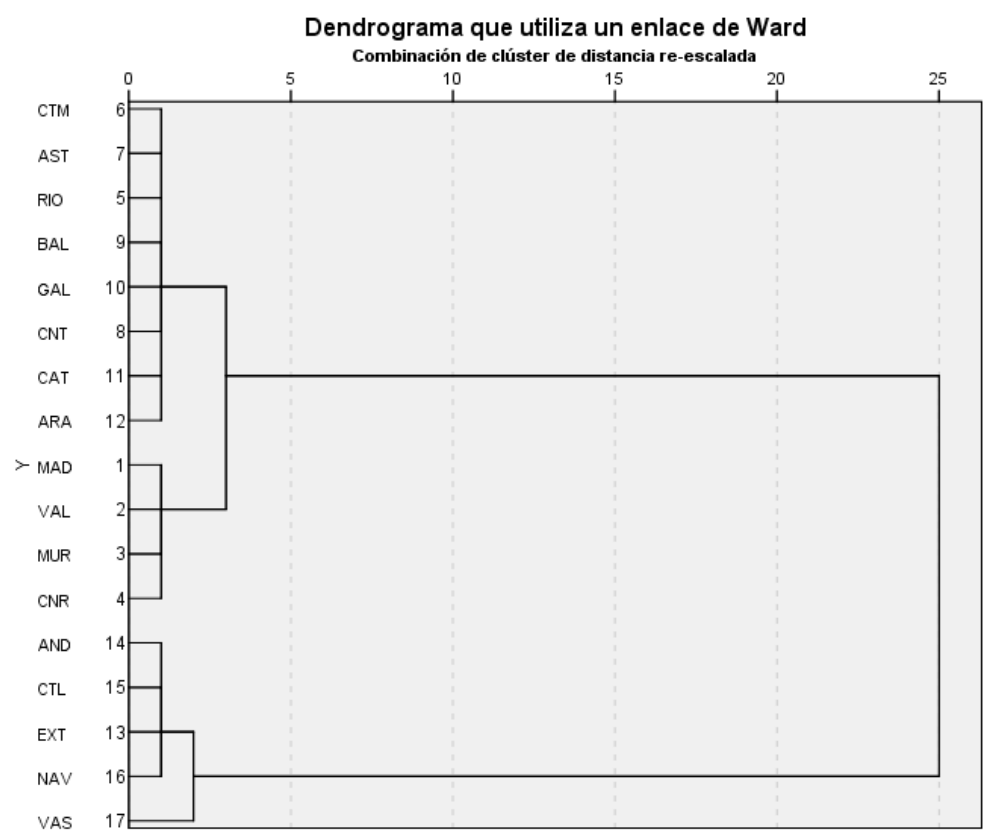

Fuente: elaboración propia.

El estudio tipológico para el indicador TOD propone de forma nítida la división en dos grupos: nueve autonomías con cero puntos (Castilla-La Mancha, Canarias, Cantabria, Comunidad de Madrid, Comunidad Valenciana, Galicia, La Rioja, Principado de Asturias y Región de Murcia), y ocho con puntuación positiva (Andalucía, Aragón, Baleares, Castilla y León, Cataluña, Extremadura, Comunidad Foral de Navarra y País Vasco).

Sin embargo, para el indicador TG, la tarea no parece tan sencilla, por lo que se aplica el método de Ward. La figura 2 muestra la representación gráfica o dendograma, y la tabla 5 el historial de conglomeraciones que permite observar paso a paso cómo se agrupan las distintas autonomías.

A la luz de los resultados obtenidos podríamos considerar, como soluciones válidas, la existencia de tres grupos de autonomías: en el extremo superior de la figura número 2 estarían las CC. AA. con valoraciones intermedias: Castilla-La 
Mancha, Principado de Asturias, La Rioja, Islas Baleares, Galicia, Cantabria, Cataluña y Aragón (mínimo 15 - máximo 29 puntos), en la parte central las que obtienen peores calificaciones: Comunidad de Madrid, Comunidad Valenciana, Región de Murcia y Canarias (mínimo 8 - máximo 12 puntos), y en la parte inferior las mejores: Andalucía, Castilla y León, Extremadura, Comunidad Foral de Navarra y País Vasco (mínimo 40 puntos - máximo 59 puntos). Una propuesta igualmente relevante sería afirmar la existencia de únicamente dos grupos, que se conseguiría fusionando las CC. AA. con puntuaciones inferiores e intermedias.

Tabla 5. Historial de conglomeración TG13, clúster jerárquico método de Ward

\begin{tabular}{|c|c|c|c|c|c|c|}
\hline \multirow{2}{*}{ Etapa } & \multicolumn{2}{|c|}{ Clúster combinado } & \multirow{2}{*}{ Coeficientes } & \multicolumn{2}{|c|}{ Primera aparición del clúster de etapa } & \multirow{2}{*}{ Etapa siguiente } \\
\hline & Clúster 1 & Clúster 2 & & Clúster 1 & Clúster 2 & \\
\hline 1 & 6 & 7 &, 000 & 0 & 0 & 9 \\
\hline 2 & 1 & 2 &, 000 & 0 & 0 & 10 \\
\hline 3 & 9 & 10 &, 500 & 0 & 0 & 4 \\
\hline 4 & 8 & 9 & 2,000 & 0 & 3 & 11 \\
\hline 5 & 14 & 15 & 4,000 & 0 & 0 & 8 \\
\hline 6 & 3 & 4 & 6,000 & 0 & 0 & 10 \\
\hline 7 & 11 & 12 & 10,500 & 0 & 0 & 13 \\
\hline 8 & 13 & 14 & 16,500 & 0 & 5 & 12 \\
\hline 9 & 5 & 6 & 22,500 & 0 & 1 & 11 \\
\hline 10 & 1 & 3 & 31,500 & 2 & 6 & 15 \\
\hline 11 & 5 & 8 & 55,500 & 9 & 4 & 13 \\
\hline 12 & 13 & 16 & 82,500 & 8 & 0 & 14 \\
\hline 13 & 5 & 11 & 190,875 & 11 & 7 & 15 \\
\hline 14 & 13 & 17 & 383,075 & 12 & 0 & 16 \\
\hline 15 & 1 & 5 & 743,450 & 10 & 13 & 16 \\
\hline 16 & 1 & 13 & 3783,765 & 15 & 14 & 0 \\
\hline
\end{tabular}

Fuente: elaboración propia.

El hecho de que $k$-means proponga una agrupación alternativa a Ward para la opción de tres clústeres, (centros iniciales 8,59 y 29; finales 12,71, 46,60 y 23,60), pero idéntica cuando se plantea la división de las autonomías en dos conglomerados, lo que consigue en tan solo dos iteraciones (centros iniciales en 8 y 59 , finales en 17,25 y 46,60 ) nos hace decantarnos por la existencia de dos grupos: uno rezagado compuesto por 12 autonomías y otro avanzado por cinco (País Vasco, Comunidad Foral de Navarra, Extremadura, Castila León y Extremadura), según puede observarse en la figura 3. 
Figura 3. Puntuaciones transparencia open data y transparencia global 2013



Fuente: elaboración propia.

\subsection{Resultados diciembre 2017}

La figura 4 ofrece la valoración de cada uno de los repositorios analizados durante la revisión de 2017. En el resumen ofrecido por la tabla 6 se aprecia con nitidez cómo los portales de transparencia han mejorado su desempeño relegando la aportación de la web institucional a un papel testimonial.

Las tablas 4 y 6 permiten realizar una comparativa de los resultados de 2013 respecto a los del año 2017. Especialmente relevante es que los POD han aumentado los ítems atendidos respecto a 2013 (37 versus 51), lo que sin embargo no es suficiente para recortar la diferencia frente a los PT (es más, la distancia aumenta hasta verse triplicada en número de ítems). Ello es debido a que en 2017 todas las CC. AA. disponen ya de PT con numerosa información disponible (a pesar de lo cual, y puesto que siguen descuidando los formatos, la ventaja en puntuación se limita simplemente al doble).

Pasando al análisis tipológico, debemos mencionar que el método de Ward propone (figura 5), y $k$ means confirma, que para el indicador TOD existen dos grupos de autonomías, unas más avanzadas: País Vasco, Andalucía, Cataluña, Comunidad Foral de Navarra, Castilla y León, Aragón y La Rioja, y otras más rezagadas: Islas Baleares, Extremadura, Canarias, Castilla-La Mancha, Región de Murcia, Cantabria, Comunidad de Madrid y Comunidad Valenciana. La línea divisoria entre ambos grupos se encuentra exactamente entre los 20 puntos de $\mathrm{La}$ Rioja y Aragón (las autonomías peores de las mejores) y los 10 puntos de Islas Baleares (la mejor autonomía de las peores). 
Figura 4. Aportación a transparencia open data y transparencia global por repositorio 2017

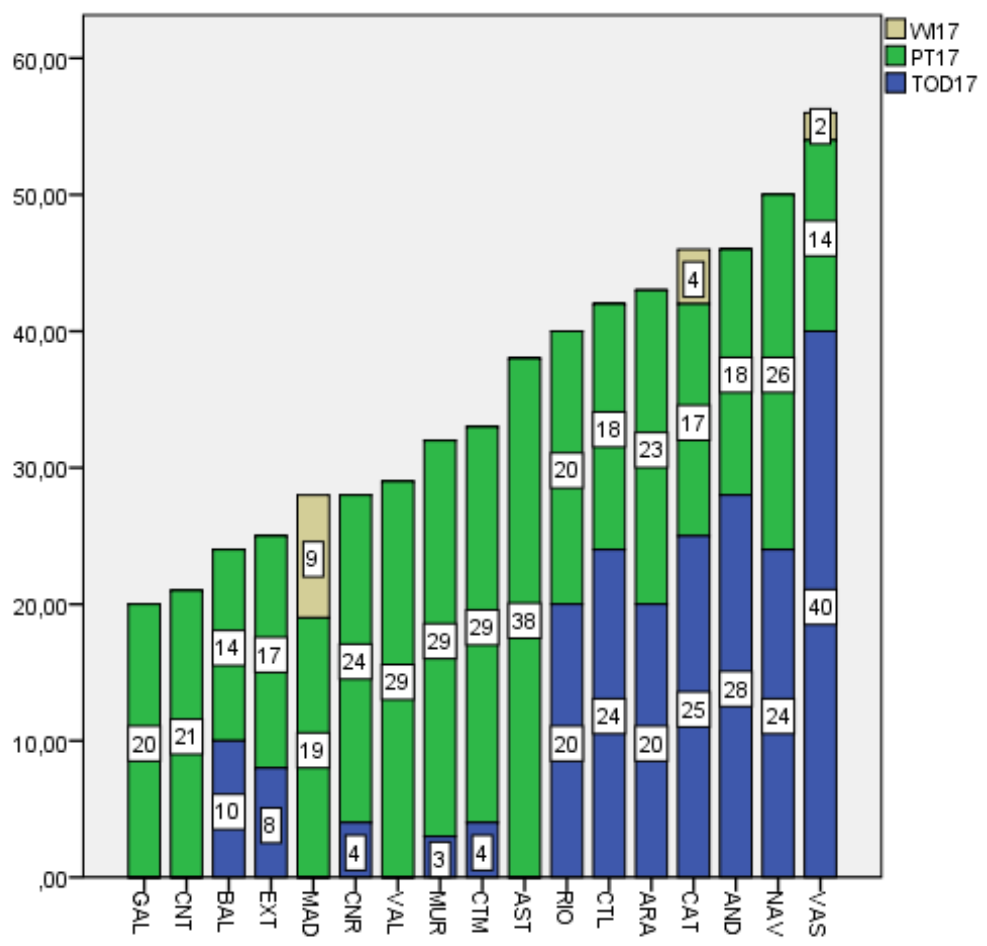

Fuente: elaboración propia.

En 2017 vuelve a ocurrir, de nuevo, que las diferencias en la puntuación alcanzadas por las CC. AA. son más reducidas para el indicador, por lo que el análisis clúster se hace imprescindible. La figura 6 muestra el dendograma propuesto por Ward, y la tabla 7 el historial de conglomeraciones.

Tabla 6. Ítems atendidos, puntos alcanzados y sus valoraciones medias 2017

\begin{tabular}{|l|c|c|c|c|}
\hline Iniciativa & Ítems atendidos & Media ítems & Puntos totalizados & Media puntos \\
\hline POD & 51 & 3,00 & 210 & 12,35 \\
\hline PT & 173 & 10,18 & 376 & 22,12 \\
\hline WI & 6 & 0,35 & 15 & 0,88 \\
\hline En conjunto & 230 & 13,53 & 601 & 35,35 \\
\hline
\end{tabular}

Fuente: elaboración propia.

Para poder decantarnos entre la existencia de dos o cuatro conglomerados aplicamos $k$ means. Puesto que, al elegir cuatro grupos, la sugerencia de 
clasificación de $k$ means $^{8}$ difiere de lo obtenido por Ward, nos quedamos con la solución de dos clústeres ya que este caso la asignación autonómica de ambos métodos es coincidente. De esta forma, tendríamos un grupo de nueve autonomías rezagadas (Castilla-La Mancha, Región de Murcia, Comunidad Valenciana, Canarias, Comunidad de Madrid, Extremadura, Islas Baleares, Cantabria, y Galicia) y ocho avanzadas (País Vasco, Comunidad Foral de Navarra, Andalucía, Cataluña, Aragón, Castilla y León, La Rioja y Principado de Asturias).

Figura 5. Dendograma transparencia open data 2017, clúster jerárquico método de Ward

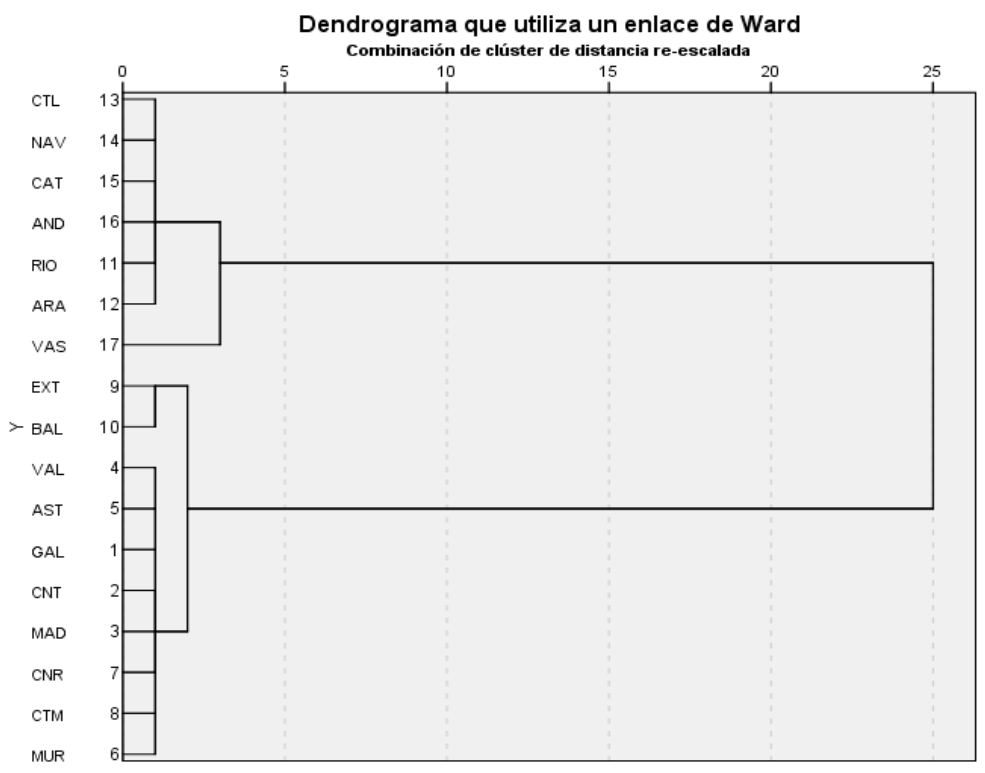

Fuente: elaboración propia.

La mayor parte de este grupo de ocho autonomías más avanzadas en TG se puede identificar claramente en la figura 7, que representa las puntuaciones de las CC. AA. según ambos indicadores. Vemos, en la parte superior derecha, a País Vasco, líder destacado, seguido por la Comunidad Foral de Navarra, Andalucía, Cataluña, Castilla y León, Aragón y La Rioja. Principado de Asturias sería la siguiente, con una aceptable puntuación en TG, pero con cero puntos en TOD, algo marcadamente singular.

8 Grupo 1: Galicia, Cantabria, Islas Baleares y Extremadura. Grupo 2: Comunidad de Madrid, Canarias, Comunidad Valenciana y Región de Murcia. Grupo 3: Principado de Asturias, La Rioja, Castilla y León, Aragón, Cataluña y Andalucía. Grupo 4: Comunidad foral de Navarra y País Vasco. 
Figura 6. Dendograma transparencia global 2017, clúster jerárquico método de Ward

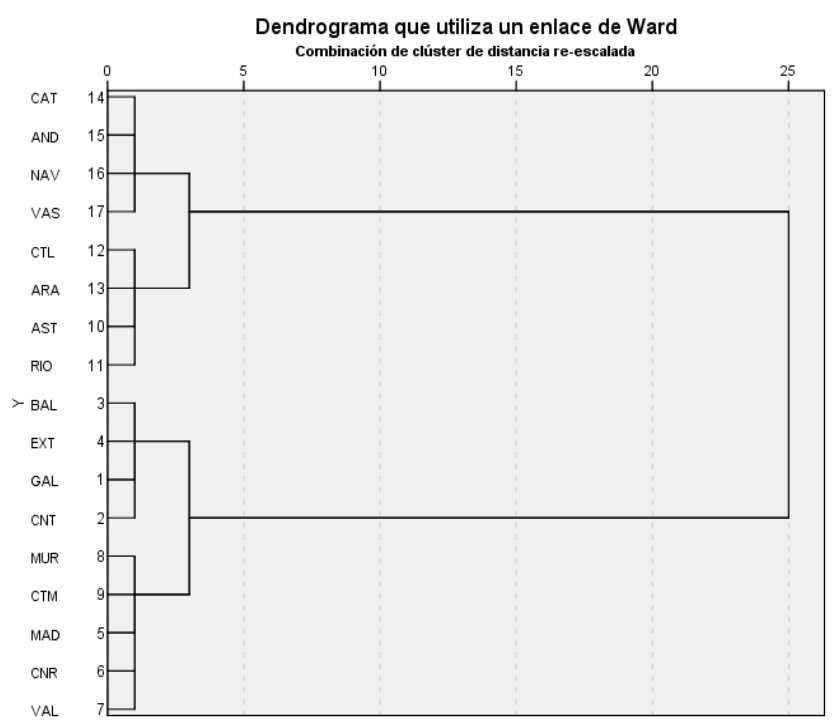

Fuente: elaboración propia.

Tabla 7. Historial de conglomeración TG17, clúster jerárquico método de Ward

\begin{tabular}{|r|r|r|r|r|r|r|}
\hline \multirow{2}{*}{ Etapa } & \multicolumn{2}{|c|}{ Clúster combinado } & \multirow{2}{*}{ Coeficientes } & \multicolumn{2}{|c|}{ Primera aparición del clúster de etapa } & \multirow{2}{*}{ Etapa siguiente } \\
\cline { 2 - 3 } & Clúster 1 & Clúster 2 & & Clúster 1 & Clúster 2 & \\
\hline 1 & 14 & 15 &, 000 & 0 & 0 & 9 \\
\hline 2 & 5 & 6 &, 000 & 0 & 0 & 7 \\
\hline 3 & 12 & 13 &, 500 & 0 & 0 & 10 \\
\hline 4 & 8 & 9 & 1,000 & 0 & 0 & 12 \\
\hline 5 & 3 & 4 & 1,500 & 0 & 0 & 11 \\
\hline 6 & 1 & 2 & 2,000 & 0 & 0 & 11 \\
\hline 7 & 5 & 7 & 2,667 & 2 & 0 & 12 \\
\hline 8 & 10 & 11 & 4,667 & 0 & 0 & 10 \\
\hline 9 & 14 & 16 & 15,333 & 1 & 0 & 13 \\
\hline 10 & 10 & 12 & 27,583 & 8 & 3 & 15 \\
\hline 11 & 1 & 3 & 43,583 & 6 & 5 & 14 \\
\hline 12 & 5 & 8 & 64,417 & 7 & 4 & 14 \\
\hline 13 & 14 & 17 & 120,750 & 9 & 0 & 16 \\
\hline 14 & 1 & 5 & 245,750 & 11 & 12 & 16 \\
\hline 15 & 10 & 14 & 398,875 & 10 & 13 & 0 \\
\hline 16 & 1 & 10 & 1841,882 & 14 & 15 & 16 \\
\hline & & & & & & 16 \\
\hline
\end{tabular}

Fuente: elaboración propia. 
Figura 7. Puntuaciones transparencia open data y transparencia global 2017



Fuente: elaboración propia.

\section{Conclusiones, limitaciones y futuras líneas de investigación}

Es necesario, para que el concepto del gobierno abierto avance y se desarrolle con plenitud, un adecuado ejercicio de publicidad activa por parte de las Administraciones públicas, dando a conocer el destino de los fondos públicos y justificando sus actuaciones. Valorar el grado en que los repositorios digitales autonómicos españoles están cumpliendo esa función es el objetivo de este estudio, que se ha realizado en dos periodos de tiempo, 2013 y 2017, a partir de una lista de comprobación compuesta por 20 ítems, e inspirada en los artículos 6 y 8 de la Ley 19/2013 de transparencia, acceso a la información pública y buen gobierno. Una vez ponderados los formatos en que los ítems son atendidos según la escala cinco estrellas, se han formulado dos indicadores: transparencia open data, que se circunscribe en exclusiva a los portales de datos abiertos, y transparencia global, que incorpora en su búsqueda además a los portales de transparencia y a la web institucional.

Evidentemente, lo medido es solo una dimensión de la transparencia, y serán futuras investigaciones las que contemplen, además, si se está realizando una correcta atención a las solicitudes de información ciudadana e incorporen otros aspectos como las licencias de uso de la información o el fomento de la participación y colaboración ciudadana. De todas formas, creemos que es un excelente punto de partida para analizar un campo aún poco explorado, y que permite enunciar interesantes conclusiones.

Los resultados del estudio comienzan describiendo la situación general en cada periodo, que se caracteriza por valores bajos de los dos indicadores: puntuaciones 
medias de 10,4 y 13,5 puntos en 2013 y 2017 para transparencia open data, y puntuaciones medias de 25,8 y 35,3 puntos en 2013 y 2017 para transparencia global. Consideramos que son unas mediocres valoraciones, puesto que sería posible alcanzar los 100 puntos simplemente considerando la divulgación de los portales de open data. Apreciamos, además importantes diferencias sobre la aportación de cada iniciativa en función del año de estudio. En 2013 la contribución de cada repositorio es prácticamente idéntica, mientras que en 2017 los portales de transparencia se convierten en la iniciativa estrella, llegando casi a duplicar la aportación de los portales de open data y reduciendo a testimonial el papel de la web autonómica.

La realización de la clasificación tipológica autonómica es aparentemente sencilla cuando se emplea el indicador transparencia open data que, en 2013, distingue entre las autonomías con cero puntos, de aquellas con valoración positiva, y en 2017 a las que no superan los 10 puntos de las que sí. Sin embargo, la tarea es más compleja cuando se considera la valoración del indicador de transparencia global, por lo que el análisis clúster es de gran ayuda. A partir de él, en el año 2013 nos decantamos por la existencia de dos conglomerados (idénticos para Ward y k-means; pertenecen al grupo de divulgación avanzada aquellas autonomías con una valoración igual o superior a los 40 puntos. En 2017, y con distancias aún menores, nuevamente Ward y $k$ means proponen dos grupos, siendo la última autonomía avanzada Principado de Asturias con 38 puntos.

De todas formas, se aprecia un comportamiento dispar a nivel longitudinal. Se pasa de una clara polarización en 2013, donde unas comunidades autonómicas simplemente replicaban iniciativas que les aportaban modernidad (teoría neoinstitucional) y otras practicaban una mayor divulgación (teoría de la agencia), a una situación con puntuaciones más compactas, menos dispersas y más normalizadas para 2017. No obstante, a lo largo de todo el estudio, el número de conglomerados propuestos ha sido de dos.

La asignación autonómica ha sido bastante estable, aunque se han producido varias permutaciones de autonomías por grupo, en especial cuando se emplea el indicador de transparencia global. Nos referimos, por un lado, a Extremadura, quien no actualiza información y cae al grupo de las autonomías rezagadas y, por otro lado, a La Rioja, Aragón y Principado de Asturias, que ascienden al grupo avanzado gracias a su notable mejoría.

Seguiremos atentos a la evolución de los repositorios digitales que facilitan la divulgación de la información pública. Hemos asistido al proceso en que portales de datos abiertos y portales de transparencia, incipientes en 2013, se han consolidado en 2017, y en la actualidad continúan en permanente desarrollo. Deseamos que estas iniciativas sigan ayudando a vislumbrar el interior de las organizaciones, su desempeño y su funcionamiento, contribuyendo a un mejor desarrollo del gobierno abierto, aunque todavía quede mucho margen de mejora, ya que los valores de los indicadores son bajos. Para ello es necesario que los portales de transparencia mejoren sus posibilidades de reutilización y los de datos abiertos incorporen más información asociada con la rendición de cuentas. 


\section{Bibliografía}

Abascal Fernández, E. y I. Grande Esteban (1994): Aplicaciones de investigación comercial, Esic Editorial, Madrid.

Abascal Fernández, E. y I. Grande Esteban (2007): Fundamentos y técnicas de investigación comercial, Esic Editorial, Madrid ( $9^{\mathrm{a}}$ edición).

Alcaide Muñoz, L., M. P. Rodríguez Bolívar y C. L. Villamayor Arellano (2019): “Open Government Initiatives in Spanish Local Governments: An Examination of the State of the Art", Governance Models for Creating Public Value in Open Data Initiatives, Springer, Cham, pp. 123-139.

Anderberg, M. R. (1973): Cluster Analysys por Applications, Academic Press, Nueva York.

Bakar N. B. y Z. Saleh (2011): "Incentives for disclosure of accounting information in public sector: a literature survey", International Research Journal of Finance and Economics, 75(1), pp. 24-38.

Bastida, F., B. Benito, M. D. Guillamón, y A. M. Ríos (2019): “Tax mimicking in Spanish municipalities: expenditure spillovers, yardstick competition, or tax competition?", Public Sector Economics, 43(2), pp. 115-139. http://dx.doi.org/10.3326/pse.43.2.1

Bauhr, M. y M. Grimes (2012): "What is transparency Government? the Quality of Government", Gothenburg, University of Gothenburg.

Bellod-Redondo, J. F. (2008): "Significado y evolución de la transparencia presupuestaria en las Comunidades Autónomas", Auditoría pública, (45), pp. 103-115.

Beltrán-Orenes, P. y E. Martínez-Pastor (2016): "Grado de cumplimiento de las leyes de transparencia, acceso y buen gobierno y de reutilización de los datos de contratación de la administración central española", El profesional de la información, 25(4), pp. 557-567.

Beltrán-Orenes, P. y E. Martínez-Pastor (2019): "Publicidades activa y pasiva en contratación pública. Una panorámica autonómica española”, El profesional de la información, 28(3), pp. 1-12. https://doi.org/10.3145/epi.2019.may.19

Berners-Lee, T. (2010): Linked Data, World Wide Web Consortium. Disponible en: https://www.w3.org/DesignIssues/LinkedData.html [Consulta: 31 de diciembre de 2019].

Boix, A. (2015): "Transparencia, participación y procedimiento en la elaboración de disposiciones reglamentarias para un modelo de open government", en: Cotino-Hueso, L., J-L. Sahuquillo-Orozco y L. Corredoira-Alfonso (eds.), El paradigma del gobierno abierto. Retos y oportunidades de la participación, transparencia y colaboración. Madrid, Universidad Complutense de Madrid, pp. 123-129.

Calderón, C. y S. Lorenzo (2010): Open Government: Gobierno Abierto, Jaén, Algón editores.

Campos Acuña, C. y A. Vaquero García (2019): "El portal de transparencia local de Galicia como ejercicio de transparencia desde la administración autonómica y local", Revista española de la transparencia, 8(3), pp. 121-135.

Cerrillo-Martínez, A. (2012): "La contribución de las TIC a la mejora de la transparencia administrativa", Arbor, 188(756), pp. 707-724.

https://doi.org/10.3989/arbor.2012.756n4006 
Cerrillo-Martínez, A. (2014): "Los principios de datos abiertos en la legislación española", Revista de internet, derecho y politica, 19, pp. 62-77.

Cerrillo-Martínez, A. (2018): "Datos masivos y datos abiertos para una gobernanza inteligente", El profesional de la información, 27(5).

https://doi.org/10.3145/epi.2018.sep.16

Chapman, R. A. y M. Hunt (1987): Open Government. A study of the prospects of open government within the limitations of the British political system, London, Routledge.

Cobo, C. (2013): "Gobierno abierto: de la transparencia a la inteligencia cívica", en Ramírez-Alujas, Á., A. Hofmann y J. A. Bojórquez Pereznieto (coords.), La promesa del Gobierno Abierto (pp. 101-118). México: ITAIP e InfoDF. http://www.itaip.org.mx/slide/pdf/lpga.pdf

Conejero Paz, E. (2013): "Gobierno abierto y democracia participativa", $3 c$ Empresa: investigación y pensamiento crítico, 2(4).

Cordis, A. S. y P. L. Warren (2014): "Sunshine as disinfectant: The effect of state Freedom of Information Act laws on public corruption”, Journal of public economics, 115, pp. 18-36.

Cotino-Hueso, L. (2013): “Derecho y 'gobierno abierto'. La regulación de la transparencia y la participación y su ejercicio a través del uso de las nuevas tecnologías y las redes sociales por las Administraciones públicas: Propuestas concretas", Revista Aragonesa de Administración Pública, 14, pp. 51-92.

Cotino-Hueso, L. (2015a): "Derechos humanos, internet y TICs", en: Rey-Martínez, Fernando (dir.), Los derechos humanos en España: un balance crítico, Valencia, Tirant Lo Blanch, pp. 449-513.

Cotino-Hueso, L. (2015b): La nueva Ley de transparencia y acceso a la información, Universidad de Alcalá servicio de publicaciones, Madrid. Disponible en:

http://hdl.handle.net/10017/22075

[Consulta: 31 de diciembre de 2019].

Criado Grande, J. I. (2013): "Redes sociales para unas administraciones y gobiernos abiertos. Desafíos para la innovación y la creación de valor público", Trabajo presentado en XVIII Congreso Internacional Del CLAD Sobre La Reforma Del Estado y De La Administración Pública, Montevideo, Uruguay, 29 de octubre al 1 de noviembre.

Curto-Rodríguez, R. (2015): "Los portales de datos autonómicos y la rendición de cuentas", Auditoría pública, 66, pp. 75-83. Disponible en: http://asocex.es/los-portales-de-datos-abiertos-autonomicos-y-la-rendicion-de-cuentas

Curto-Rodríguez, R. (2017): Datos abiertos y rendición de cuentas en las comunidades autónomas españolas. Niveles de divulgación y determinantes, tesis doctoral, Universidad de Oviedo, Departamento de Contabilidad. Disponible en: http://digibuo.uniovi.es/dspace/bitstream/10651/42797/1/TD_ricardocurto.pdf

Curto-Rodríguez, R. (2020): "Transparencia operativa de las comunidades autónomas españolas mediante sus portales de datos abiertos", El profesional de la información, 29(1). https://doi.org/10.3145/epi.2020.ene.15

Delgado Morales, F., F. J. López Carvajal y J. Sierra Rodríguez (2015): "Regulación y Sistemas de Evaluación de la Transparencia en España", Revista de derecho de la Hacienda Pública, 5, pp. 111-134. 
Esteller-Moré, A. y J. Polo Otero (2012): “Fiscal Transparency. (Why) does your local government respond?", Public Management Review, 14(8), pp. 1153-1173.

Everitt, B. S., S. Landau y M. Leese (2001): Cluster Analysis (Cuarta Edición), Editorial Arnold, Londres.

Fariña Gómez, B., J. Fernández de la Mora y H. Fernández-Abascal Teira (1996): "Desigualdades territoriales en el bienestar. Clasificación jerarquizada de las provincias españolas mediante un análisis clúster con semillas predeterminadas", Anales de estudios económicos y empresariales 11, pp. 469-490.

Ferejohn, J. (1999): "Accountability and authority: towards a model of political accountability", en: Przeworski, A., Stokes, S. C. y Manin, B. (eds.), Democracy, Accountability and Representation (pp. 131-153), New York, Cambridge University Press.

Fernández Llera, R. (2015): “Transparencia y rendición de cuentas locales tras la supuesta racionalización”, Presupuesto y Gasto Público, 81, pp. 131-150.

Fernández Llera, R. y E. Morán Méndez (2013): "Las Comunidades Autónomas en la crisis: cifras, planes y retos de control", Auditoría pública: revista de los Órganos Autónomos de Control Externo, 59, pp. 39-50.

Ferrán Aranaz, M. (1996): SPSS para Windows: Programación y análisis estadístico, Editorial McGraw-Hill, Madrid.

García-García, J. y R. Curto-Rodríguez (2019): "El ejercicio de la rendición de cuentas mediante portales de datos abiertos en las comunidades autónomas españolas", IDP, Revista de internet, derecho y política, 29. https://doi.org/10.7238/idp.v0i29.3182

Giménez-Chornet, V. (2012): “Acceso de los ciudadanos a los documentos como transparencia de la gestión pública", El profesional de la información, 21(5), pp. 504-508. https://doi.org/10.3145/epi.2012.sep.09

Gomez Macfarland, C. A. (2016): "Las cuatro direcciones de la transparencia y el servicio de administración tributaria: un estudio de caso", Buen Gobierno, 21(5), pp. 107-132.

Guichot Reina, E. (2014): Transparencia, acceso a la información pública y Buen Gobierno, Estudio de la Ley 19/2013, de 9 de diciembre, Editorial Tecnos, Madrid.

Hair Jr, J. F., R. E. Anderson, R. L. Tatham y W. C. Black (1999): Análisis Multivariante ( $5^{\mathrm{a}}$ edición), Prentice Hall Ibérica, Madrid.

Höchtl, J. (2012): "Open Government Data-Beyond the Hype", en CeDEM 12 Conference for E-Democracy and Open Government, Danube-University Krems, Austria, EditionDonau-Univ, Krems, pp. 363-366.

Johnson, D. E. (1998): Apllied Multivariate Methods for Data Analysys, Brooks Cole Publising Company, Nueva York.

Krah, R. y G. Mertens (2020): "Democracy and financial transparency of local governments", en Sub-Saharan Africa, Meditari Accountancy Research. https://doi.org/10.1108/MEDAR-08-2019-0539

Laswad, F., R. Fisher y P. Oyelere (2005): "Determinants of voluntary Internet financial reporting by local government authorities", Journal of Accounting and Public Policy, 24(2), pp. 101-121.

Lévy, J. P. y J. Varela Mallou (eds). (2003): Análisis multivariable para las Ciencias Sociales, Madrid, Pearson Educación, S.A.

Lizcano, J. (2016): "Transparencia y prevención de la corrupción: El rol de la sociedad civil”, La corrupción en España, Lapuente, V. (coord.), Madrid, Alianza Editorial. 
Luque Martínez, T. (2000): Técnicas de análisis de datos en investigación de mercados, Editorial Pirámide, Madrid.

Mariñez Navarro, F. (2013): "El debate del gobierno abierto", Buen Gobierno, 14(3), pp. 39-64.

Mariñez Navarro, F. (2017): “Completar y complementar en la era de la digitalización. La poliarquía plus como contexto”, Buen Gobierno 22(5), pp. 48-62.

Martín Martín, Q. y Y. R. de Paz Santana (2007): Tratamiento estadístico de datos con SPSS, Editorial Paraninfo.

Martínez Miranda, M. D. (2000): Análisis Cluster en SPSS. Disponible en: http://www.ugr.es/ curspss/archivos/Cluster/cluster.pdf [Consulta: 31 de diciembre de 2019].

Martínez Moya, D. (2015): "Evaluación de los aspectos de usabilidad y reutilización de información en el Portal de la Transparencia de España", Cuadernos de gestión de información, 5, pp. 36-52.

Masip, P., C. Ruiz-Caballero y J. Suau. (2019): “Active audiences and social discussion on the digital public sphere. Review article", El profesional de la información, 28(2). https://doi.org//10.3145/epi.2019.mar.04

Obama (2009): Memorandum for the Heads of Executive Departments and Agencies: Transparency and Open Government, Washington, DC. Disponible en: https://www.whitehouse.gov/the_press_office/TransparencyandOpenGovernment [Consulta: 31 de diciembre de 2019].

Parks, W. (1957): "Open Government Principle: Applying the right to know under the Constitution", George Washington Law Review., 26(1), pp. 1-77.

Pérez López, C. (2005): Métodos estadísticos avanzados con SPSS, Editorial Paraninfo, Madrid.

Reddick, C. G. (2011): "Citizen interaction and e-government: Evidence for the managerial, consultative, and participatory models", en Transforming Government: People, Process and Policy, 5(2), 167-184. https://doi.org/10.1108/17506161111131195

Salvador Figueras, M. (2001): "Análisis de conglomerados o clúster", Disponible en: http://www.5campus.org/leccion/cluster [Consulta: 31 de diciembre de 2019].

Sánchez De Diego Fernández De La Riva, M. (2014): "El día después de la Ley de transparencia", Revista jurídica de Castilla y León, 33, pp. 12-20.

Sharma, S. (1996): Apllied Multivariate Tecchniques, Wiley\&Sons, Nueva York.

Sierra-Rodríguez, J. (2018a): "Apuntes básicos sobre la Ley de Transparencia, Acceso a la Información Pública y Buen Gobierno", en Sánchez de Diego Fernández de la Riva, M. Apuntes sobre la Transparencia, Madrid, UCM.

Sierra-Rodríguez, J. (2018b): "Una crítica al diseño de los organismos de garantía del derecho de acceso en las Comunidades Autónomas", Revista jurídica de Castilla y León, (45), pp. 73-112.

Trespalacios Gutiérrez, J. A., L. Bello Acebrón y R. Vázquez Casielles (2005): Investigación de mercados: Métodos de recogida y análisis de la información para la toma de decisiones en marketing, Editorial Paraninfo, Madrid.

Trespalacios Gutiérrez, J. A., R. Vázquez Casielles, F. J. de la Ballina Ballina y A. Suárez Vázquez (2016): Investigación de mercados: El valor de los estudios de mercado en la era del marketing digital, Editorial Paraninfo, Madrid. 
Uriel Jiménez, E. y J. Aldás Manzano (2005): Análisis Multivariante Aplicado: Aplicaciones al Marketing, Investigación de mercados, Economía, Dirección de Empresas y Turismo, Thomson Editores, Madrid.

Valderrey, P. (2010): SPSS 17. Extracción del conocimiento a partir del análisis de datos, Editorial RA-MA, Madrid.

Villoria Mendieta, M. (2012): "Paradojas y tensiones de la innovación: el caso del Open Government", P3T, Journal of public policies and territories, 2(1), pp. 11-14.

Villoria Mendieta, M. (2013): "El gobierno abierto como subsistema de políticas: Una evaluación desde el institucionalismo discursivo", en Ramírez-Alujas, Á., Hofmann, A., y Bojórquez Pereznieto, J. A. (coords.), La promesa del Gobierno Abierto, pp. 69-99. México, ITAIP e InfoDF. Disponible en: http://www.itaip.org.mx/slide/pdf/lpga.pdf [Consulta: 31 de diciembre de 2019].

Villoria Mendieta, M. (2014): La publicidad activa en la ley de transparencia, acceso a la información y buen gobierno: posibilidades e insuficiencias. Disponible en: http://transparencia.gencat.cat/web/.content/pdfs/governobert_1_es.pdf [Consulta: 31 de diciembre de 2019].

Ward Jr, J. H. (1963): "Hierarchical Grouping to Optimize an Objective Function", Journal of the American Statistical Association, 58, pp. 236-244. 